\title{
Back to basics
}

Neuroscience looks to Hydra for connections between the brain and behavior

The human brain is complicated. Billions of neurons are linked by trillions of connections, responsible for everything from memories to movement. Understanding those connections is no easy task, driving the search for evolutionarily conserved features from simpler brains that are easier to manipulate. Immense knowledge has been gained from working with species like mice and zebrafish, and invertebrates like Drosophila and C. elegans are proving invaluable in our understanding of how the nervous system works. But even an organism as seemingly simple as a fly or a worm has its neurological complexities. New research goes back-all the way back-to one of the very simplest animals to make a complete map of brain activity (Curr. Biol. 27, 1-13; 2017).

Following a suggestion from Sydney Brenner, Rafael Yuste's lab at Columbia University adopted the cnidarian Hydra vulgaris as a model of the nervous system. "You cannot go more basic than cnidarians because the animals below them don't have neurons," Yuste says, explaining that these animals possess the simplest "brains" one can study. Relatives of jellyfish and coral, Hydra are freshwater polyps, named for the tentacles resembling the mythical Greek beast. Rather than a formal brain and nervous system, Hydra instead have a "nerve net," a fairly evenly spaced assemblage of neurons that allows them to expand and contract, hunt prey and reproduce and, in a feat of acrobatics, somersault head-overfoot to move about. They are easy to maintain in the lab - the immortal animals are quite hardy-but have only recently become accessible for neuroscience research.

Two developments were needed, explains lead author Christophe Dupré. The first was the ability to create a transgenic Hydra

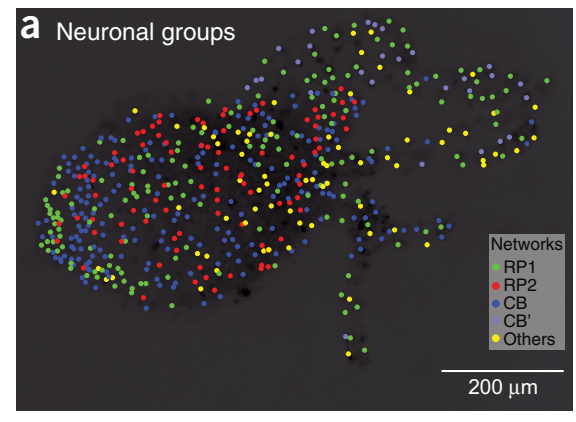

The different neuronal groups in Hydra. Image adapted from Curr. Biol. 27, 1-13; 2017.

that expresses different fluorescent markers. That happened about a decade ago, he says. They also needed an improved, higher performance calcium indicator that could capture activity at individual neurons. That came just a few years ago with the development of molecules such as GCaMP6s. Dupré and Yuste created a transgenic Hydra expressing the new indicator and, with slight modifications to their microscopy techniques, recorded neural activity simultaneously across the entire body of the millimeter long animals.

What they found were four distinct-and non-overlapping-circuits, each linked to a specific movement. One circuit elongates the animal and two others contract it radially and longitudinally, while the fourth, present below the tentacles, creates a "nodding" motion. The results suggest an answer to long-standing question about neural circuit connectivity. "Out of a tapestry of neurons that appear to be all the same and appear to be all connected, somehow evolution has been able to carve out these specific subcircuits, each of them doing a particular behavior," says Yuste.

Dupré and Yuste's work in Hydra is the first of many brain activity maps in the works as part of the Brain Activity Map project, an idea Yuste helped develop that was rebranded and expanded by President Obama as the BRAIN Initiative in 2013. The 15-year BRAIN Initiative is currently funding over 300 labs in the US and abroad to develop new neuroscience methods and techniques with the ultimate goal of imaging every spike from every neuron in the nervous system, Yuste explains. Though only a few years in, researchers involved with the Initiative are advancing imaging methods and techniques to stimulate and manipulate neuron activity; they are also improving the mathematics and statistics needed to analyze the immense amounts of data being produced and are considering how to apply all these developments to human brains. Activity maps in more traditional models, like zebrafish and Drosophila, are also advancing as the methods needed to capture neuronal activity in more complex nervous systems continue to improve.

Dupré and Yuste will continue their work in Hydra, looking next at how the different circuits coordinate more complex behavior like feeding and somersaulting. The dream is "to completely decode or reverse-engineer its brain," says Yuste, and be able to make predictions about the animals' movements.

From there, it will be a question of how those observations translate up through the evolutionary tree. Yuste expects to find similarities, and sees great potential in studying organisms he thinks have been underexploited (Trends Neurosci. 40, 92-105; 2017). Genome sequencing of cnidarians has revealed more genes than expectedHydra contains over 20,000, many of which appear to be conserved in other lineages. "The game that evolution is playing is not a game of inventing new molecules or new genes, but combining these building blocks into more complicated organisms," Yuste thinks. "That makes it really important to study these basal metazoan animals."

Ellen P. Neff 